\title{
Surgical treatment outcome of giant cell tumor of distal ulna: En bloc resection vs. curettage and bone graft
}

\author{
Khodamorad Jamshidi ${ }^{1}$, Mehrdad Bahrabadi*1, Abolfazl Bagherifard ${ }^{1}$, Mehdi Mohamadpour ${ }^{1}$
}

Received: 11 June 2017

Published: 1 June 2018

\begin{abstract}
Background: Giant cell tumor (GCT) of the bone is a benign neoplasm with local aggressive behavior. Distal ulna is a very rare place for GCT. Published studies have mainly focused on case reports, and thus there is no consistent treatment strategy for this tumor at this location. This retrospective study was conducted to evaluate the oncological and functional results of 2 different surgical treatment methods for GCT in distal ulna.

Methods: In this study, 9 patients with GCT of distal ulna were followed after surgical treatment of GCT of distal ulna. Of the patients, 2 had local recurrence after surgery in other hospitals and 7 had primarily been admitted to our hospital. Four patients, all with grade 2 Enneking and Campanacci's classification, were treated by extended curettage and bone grafting, while 5 patients with grade 3 were managed by distal ulnar resection. Minimum follow-up time was 24 months.

Results: Patients with grade 3 GCT of distal ulna, who had extended curettage, had a local recurrence up to one year after surgery. Local recurrence was never seen in those with grade 3 disease with resection or in those with grade 2 disease with extended curettage as the treatment of GCT in this location.

Conclusion: Based on our study, which is the largest cohort of GCT of distal ulna, extended curettage, and en bloc resection can be suggested as valuable methods of treatment for grades 2 and 3 tumors, respectively.
\end{abstract}

Keywords: Giant cell tumor, Ulna, Extended curettage, En bloc resection, Benign aggressive

Copyright@ Iran University of Medical Sciences

Cite this article as: Jamshidi Kh, Bahrabadi M, Bagherifard A, Mohamadpour M. Surgical treatment outcome of giant cell tumor of distal ulna: En bloc resection vs. curettage and bone graft. Med J Islam Repub Iran. 2018(1 June);32:44. https://doi.org/10.14196/mjiri.32.44

\section{Introduction}

Giant cell tumor (GCT) of the bone is an uncommon benign neoplasm with locally aggressive behavior, encompassing about $5 \%$ of all primary bone tumors (1). It usually occurs in the epiphysis of long tubular bones. The most common locations for this tumor in decreasing order of frequency are distal femur, proximal tibia, distal radius, and proximal humerus. Distal ulna is a very unusual place for GCT of the bone, encompassing about $0.45 \%$ to $3.2 \%$ of all the cases of GCTs (2).

Curettage with packing and resection with or without reconstruction of distal ulna are proposed treatment options for GCT of the distal ulna. The present study was conducted to describe the oncological and functional results of the treatment of this tumor in this rare location. To

Corresponding author: Dr Mehrdad Bahrabadi, mehrdad.bahrabadi@gmail.com

1. Bone and Joint Reconstruction Research Center, Shafa Orthopedic Hospital, Iran University of Medical Sciences, Tehran, Iran. the best of our knowledge, this was the largest case series ever published solely on GCT of the distal ulna.

\section{Methods}

In this cross-sectional study, 286 patients with confirmed GCT were surgically treated in Shafa orthopedic hospital during 1990 to 2014. However, GCT of the distal ulna was identified in 9 patients (3.5\%). Demographic information, surgical details, and histopathology reports were reviewed. No patient was excluded from the study. Staging of the tumors was determined using anteroposterior radiographs of the forearm and the chest, chest CT scan, and MRI of the forearm. Moreover, basic laboratory panel including calcium, phosphorus, alkaline phos-

$\uparrow$ What is "already known" in this topic:

GCT is an aggressive bone tumor that needs surgical treatment. As distal ulna is a very rare location for this tumor, there is no universally accepted method of treatment.

$\rightarrow$ What this article adds:

For GCT of distal ulna, extended curettage with bone grafting is a better treatment modality when it is confined to the bone. However, when the tumor breaches the cortex, we recommend tumor resection without reconstruction. 


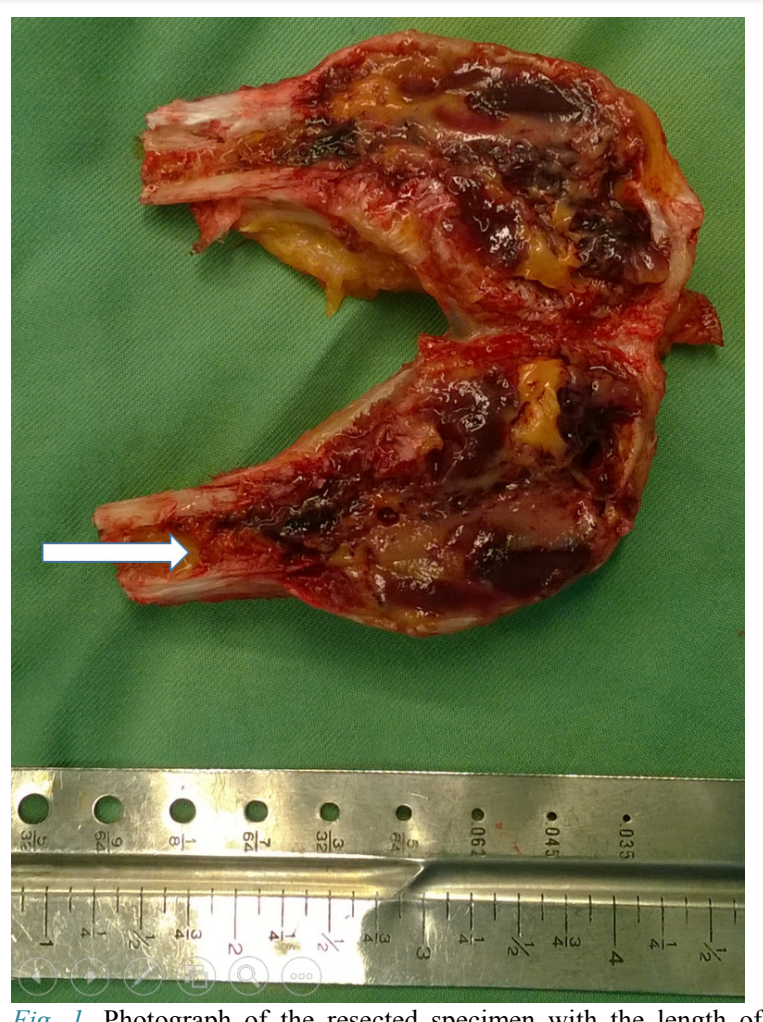

Fig. 1. Photograph of the resected specimen with the length of about 7 centimeters. The arrow shows the proximal extent of the tumor.

phatase, and PTH was used to rule out the brown tumor of hyperparathyroidism. The Enneking and Campanacci's classification was used to grade the tumors. Considering the location of palpable mass, core needle biopsy was performed for all patients from volar or dorsal side, except for 2 patients (cases number 3 and 4) who had a recurrent GCT of distal ulna after curettage and bone grafting in another hospital; they were diagnosed with histopathology at the time of admission to our center.

Patients were treated either by en bloc resection of the distal ulna (cases 3-6, and 9) or curettage and bone grafting (cases 1,2,7, and 8). In patients with distal ulna resection, a direct longitudinal approach was selected depending on the biopsy site.

An appropriate length of ulna with a bony margin of at least $10 \mathrm{~mm}$ was resected, as planned preoperatively from the MRI and biopsy tract. Distally and circumferentially, the plan of resection included the triangular fibrocartilage complex, the ulnar edge of the pronator quadratus muscle, and the capsule of distal radio-ulnar joint (Fig. 1).

After resection of the tumor, joint capsule was sutured to the remaining soft tissue. For the cases managed by curettage and bone grafting, a large window was made over the thinnest cortex. After removal of the tumor by cruet, the wall of cavity was enlarged at least $1 \mathrm{~mm}$ by high speed burring. We also employed phenol 5\% for 3 minutes for local adjuvant therapy, and the defect was filled with morselized allograft (Fig. 2).

Long arm splint was used after surgery with wide resection of distal ulna in all patients. The splint was removed at 2 weeks in those patients with curettage and bone grafting and at 4 weeks for those with distal ulnar resection. Range of motion exercises started right after splint removal. Follow-ups were scheduled at 2 and 4 weeks and at 3, 6 , and 12 months, and yearly thereafter. Physical examination was done in each follow-up visit, and a plain radiography of the forearm and chest were ordered. The oncological result at the last follow-up was evaluated for the surgical margin obtained intraoperatively, the presence of any local recurrence, evidence of pulmonary metastasis visible on anteroposterior, and lateral plain radiographies of the chest. Functional outcome was assessed based on Musculoskeletal Tumor Society Score (MSTS)(3) and the Persian Version of the Shortened Form of Disabilities of
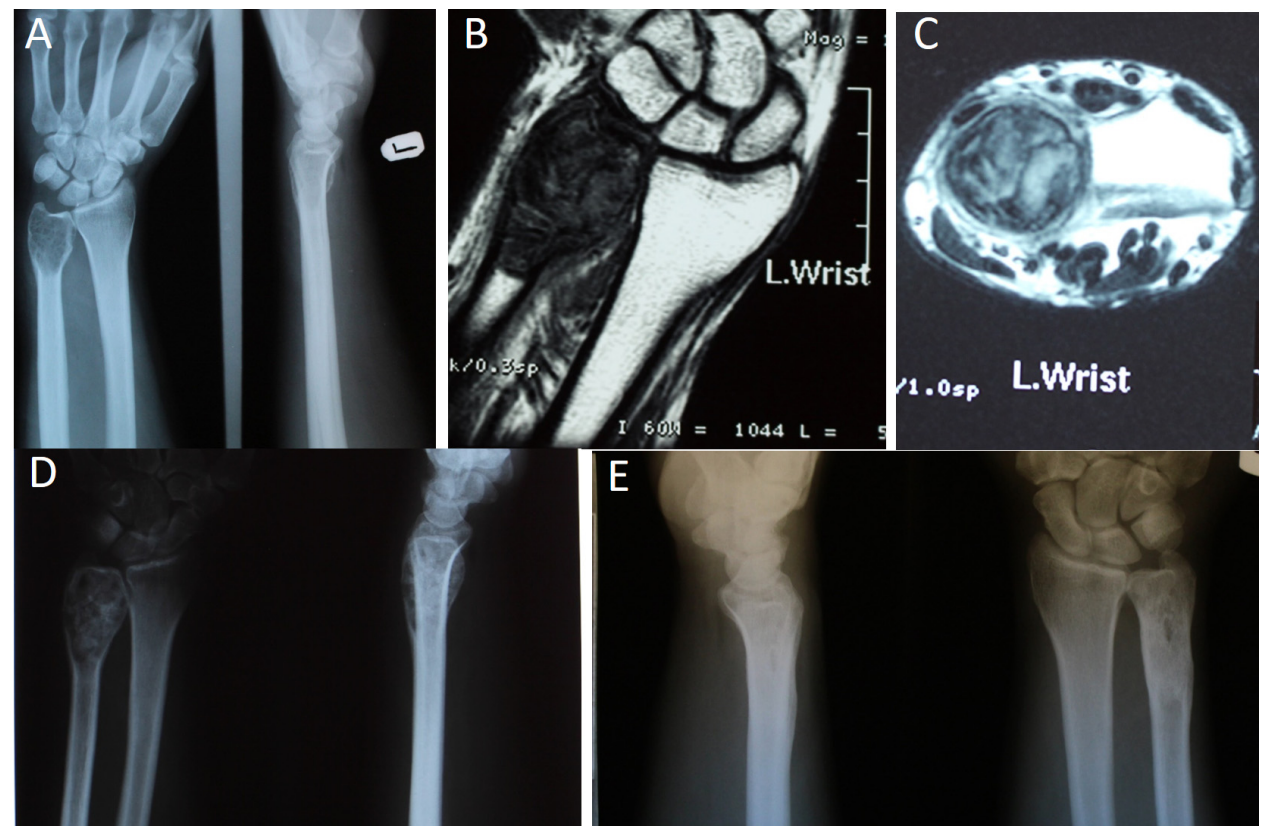

Fig. 2. A: Preoperative anteroposterior radiographs; B: Preoperative coronal T1 weighted magnetic resonance image; C: Preoperative axial T2 weighted image; D: Early postoperative radiograph 4 weeks after surgery); E: Postoperative radiograph of the patient 8 years after surgery, showing complete healing without recurrence. 
the Arm, Shoulder, and Hand (Quick-DASH) Questionnaire (4) at the latest follow- up. Written consent was obtained from all patients to publish the results of their treatment, and the study was approved by the ethical committee of our center. For statistical analysis, SPSS Version 22 (SPSS Inc, Chicago, IL, USA) was used. Descriptive statistics were presented as frequencies (\%), mean \pm standard deviation (SD) with ranges as appropriate.

\section{Results}

There were 4 males and 5 females with the average \pm SD age of $32.1 \pm 13$ years (range 19 to 65 ). Table 1 demonstrates the basic demographic details. The presenting symptom included pain for all patients. Moreover, 7 patients had swelling in addition to pain at the time of presentation. According to Enneking and Campanacci's classification, 4 patients had stage 2 disease and the remaining 5 patients had stage 3 . None of the patients had a metastatic disease at the time of diagnosis.

Patients with recurrent disease had a curettage and bone grafting as the treatment of their stage 3 Enneking and Campanacci's tumor in other hospitals, and both of them were referred to our center due to recurrence of their tumors. The time for recurrence was 7 and 9 months.

Patients with stage 3 disease were treated by wide resection of the distal segment of the ulna with no attempt for reconstruction (Fig. 3). The mean \pm SD length of the resected segment of the ulna was $6 \pm 1.45 \mathrm{~cm}$ (range 4.5 to 8 $\mathrm{cm})$.

All patients completed the follow-ups. The mean \pm SD follow-up was $68.44 \pm 28.6$ months (range 25 to 21 months). Oncological results were assessed for surgical margins obtained during surgery, local recurrence, and distant metastasis. There was no local recurrence, or any distant metastasis for the 5 patients with stage 3 who were treated by en bloc resection and for the remaining 4 patients with stage 2 who were treated by extended curettage

\begin{tabular}{|c|c|c|c|c|c|c|c|c|c|c|c|}
\hline No & Age/gender & $\begin{array}{c}\text { Dominant } \\
\text { side }\end{array}$ & $\begin{array}{c}\text { Side of } \\
\text { involvement }\end{array}$ & $\begin{array}{c}\mathrm{E} / \mathrm{C} \\
\text { grading }\end{array}$ & Biopsy & Treatment & $\begin{array}{l}\text { Amount } \\
\text { of bone } \\
\text { resection }\end{array}$ & $\begin{array}{l}\text { Follow- } \\
\text { up/months }\end{array}$ & MSTS $\%$ & $\begin{array}{l}\text { Dash } \\
\text { score }\end{array}$ & Complications \\
\hline 1 & $29 / \mathrm{M}$ & RT & $\mathrm{LT}$ & II & $\mathrm{CNB}$ & C\&BG & No & 121 & 93.3 & 2.5 & No \\
\hline 2 & $19 / \mathrm{F}$ & RT & LT & II & $\mathrm{CNB}$ & $\mathrm{C} \& \mathrm{BG}$ & No & 99 & 96.6 & 1.7 & No \\
\hline 3 & 28/M & RT & RT & III & $\mathrm{Re}$ & $\mathrm{R}$ & 5 & 56 & 86.6 & 5 & No \\
\hline 4 & 29/M & RT & RT & III & $\mathrm{Re}$ & $\mathrm{R}$ & 5.5 & 72 & 93.3 & 2.5 & No \\
\hline 5 & $27 / \mathrm{F}$ & RT & LT & III & $\mathrm{CNB}$ & $\mathrm{R}$ & 8 & 79 & 83.3 & 5.8 & Click \\
\hline 6 & 29/M & RT & LT & III & $\mathrm{CNB}$ & $\mathrm{R}$ & 7 & 25 & 90 & 3.3 & Click \\
\hline 7 & $65 / \mathrm{F}$ & RT & LT & II & CNB & C\&BG & No & 54 & 93.3 & 2.5 & No \\
\hline 8 & $36 / \mathrm{F}$ & RT & RT & II & $\mathrm{CNB}$ & C\&BG & No & 43 & 90 & 3.3 & No \\
\hline 9 & $27 / \mathrm{F}$ & RT & RT & III & $\mathrm{CNB}$ & $\mathrm{R}$ & 4.5 & 67 & 83.3 & 5.8 & Click \\
\hline
\end{tabular}
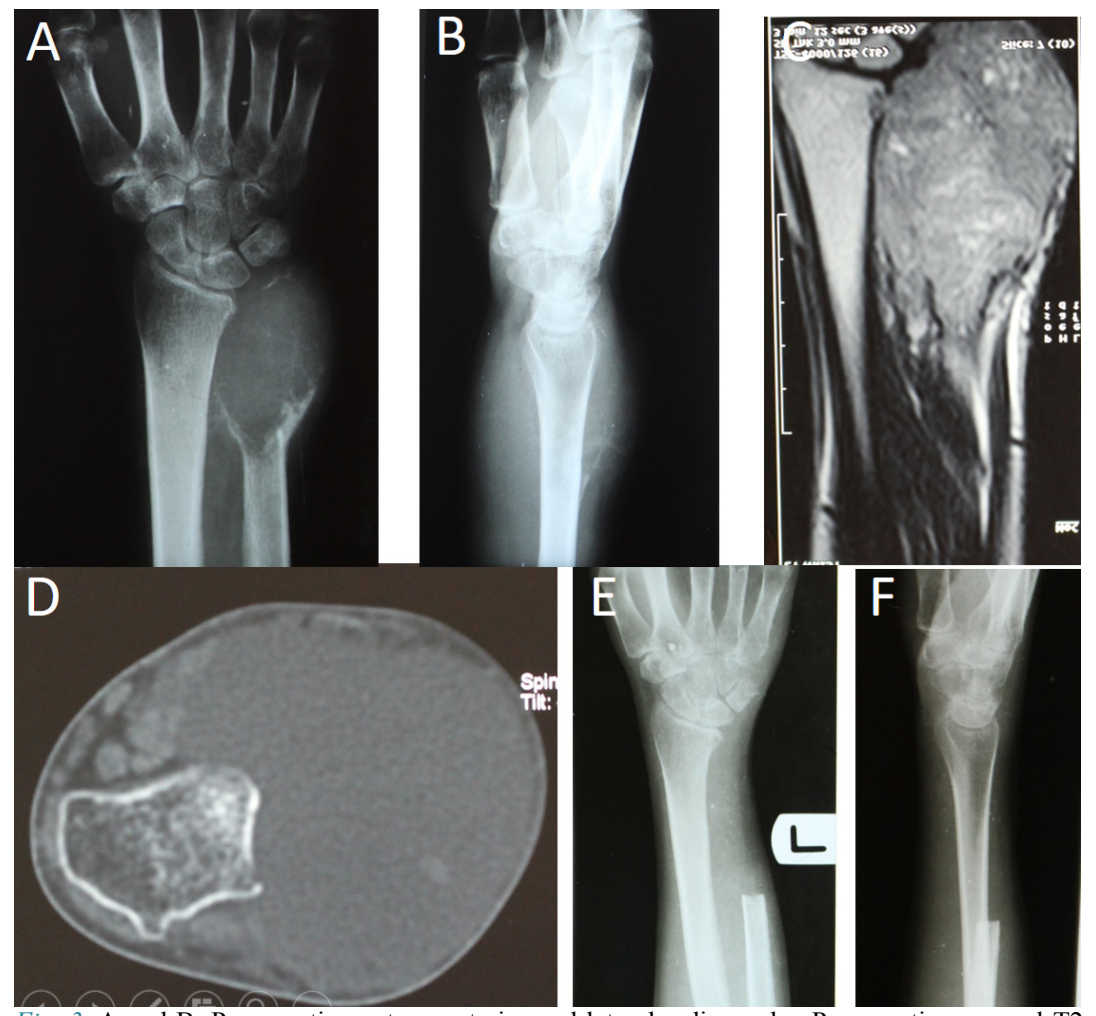

Fig. 3. A and B: Preoperative anteroposterior and lateral radiographs; Preoperative coronal T2 weighted magnetic resonance image; D: Preoperative axial computerized tomographic scan; E and $\mathrm{F}$ : Postoperative anteroposterior and lateral radiographs of the patient 6 years after surgery. 
Table 2. Oncological results as compared to the type of surgery and grade of tumor

\begin{tabular}{lcc}
\hline Surgery & Grade II & Grade III \\
\hline Resection & 0 patients & No recurrence \\
Extended Curettage and BG & No recurrence & $100 \%$ Recurrence \\
\hline
\end{tabular}

and grafting at our center (Table 2). At the latest followup, the mean \pm SD MSTS score for those patients who underwent ulnar resection was $87.3 \% \pm 4.3(83.3 \%$ to $93.3 \%)$ and the mean \pm SD DASH score was $4.48 \pm 2.1$ for distal ulna resection (5.8 to 2.5).

The mean \pm SD MSTS score for another group of the patients treated by curettage and bone graft was $93.3 \% \pm 2.7$ (90\% to $96.6 \%$ ), and the mean \pm SD DASH score was $2.5 \pm 1.3$ (range 1.7 to 3.3 ) (Table 1). Range of motion was equal to the opposite side in those patients treated by en bloc resection both in supination, pronation, and flexion and extension planes. In those with curettage and grafting, range of motion was near normal in the supination pronation and in extension flexion of the wrist compared to the opposite side. The greatest decrease in the range of motion was loss of $15^{\circ}$ in 5 supinations and $20^{\circ}$ in extension. No patient demonstrated any wrist instability, ulnar subluxation, ulnocarpal translocation, or ulnar angulation of the wrist. Although there was no instability of the carpus, 3 patients with distal ulnar resection reported occasional clicking of the wrist. There was no damage to the sensory branch of ulnar nerve in these series.

\section{Discussion}

In the treatment of GCT, the goals are to prevent local recurrence with adequate resection and preserve limb function. Thus, when the architecture of the bone and joint is preserved, intralesional curettage is the procedure of choice for this benign aggressive lesion. Many believe this recurrent benign lesion should be treated with repeat curettage. When it comes to distal end of the ulna, which is an expendable bone, there is no consensus about the best treatment due to its rarity. Distal ulna helps with forearm rotation. It also facilitates grip strength and maintains the relationship between the carpus and the distal part of the radius. The ulnar collateral ligament of the carpus and the triangular fibrocartilage complex attaching to ulnar styloid process play an important role in the maintenance of this relationship (5). There are 2 treatment options to treat benign aggressive tumors of distal ulna like GCT. Curettage and packing is the first treatment option and is used when GCT is limited to bone cortex. Resection of the distal ulna is another option and is used when the tumor is not confined to the cortices, or in case of soft tissue component, pathologic fracture, or unsuccessful previous surgery. In our study, we specifically evaluated the results of surgical treatment of distal end of ulna GCT and compared the functional outcomes of the 2 different methods of treatment. The mean MSTS score for the patients treated by curettage and bone graft was $93.3 \%$, and the mean DASH score for this group of patients was 2.5. The mean MSTS score for the patients treated by en bloc resection was $87.3 \%$ and the mean DASH score for this group of patients was 4.48. Reduced grip strength and limitation of motion of the wrist were the main cause of the inferior results. Resection of the distal ulna in the form of a long segment of bone is an accepted treatment for tumors of distal ulna. There are some potential problems with this procedure including downfall of the bony support for the triangular fibrocartilage complex, ulnocarpal joint instability leading to ulnocarpal translocation, and decreased grip strength, and unsteady rotation of the carpal unit around the ulnar axis. In addition, distal part of the remaining ulnar diaphysis may become close enough to the radius to cause a painful snapping or tendinous rupture (6). Thus, preservation of the TFCC and ulnar collateral ligament and minimum possible size resection are proposed to decrease problems associated with distal ulnar resection (6). Reconstruction of the distal radio-ulnar joint was suggested in some case series for tumors of the distal part of the ulna (6-9); these include metal prosthesis or using bone graft to reconstruct the missing segment of the distal ulna. Some authors believe that stabilization of the distal ulna is an alternative procedure to reconstruct the distal radio-ulnar joint (10). Reconstruction of the distal radio-ulnar joint includes tenodesis procedures involving extensor carpi ulnaris, flexor carpi ulnaris, or using local soft tissues or muscles to stabilize the ulnar stump (7). We believe that reconstruction with prosthesis, bone allograft, autografts, or cement causes unnecessary risk, without any obvious functional gain. In this study, 5 patients underwent en block resection. The average length of the resected segment of the ulna was $6 \mathrm{~cm}$ (range 4.5 to $8 \mathrm{~cm}$ ), in which more extensive ulnar resection than classic Darrach procedure was made, and none of the patients had ulnocarpal translocation. Moreover, when the distal end of the ulna, as an expendable bone, is resected for oncological purpose, excision of the neoplasm is more important than restoration of function. In addition, during tumor resection, it may not be possible to preserve periosteal sleeve due to oncological reasons. Denosumab, which is a human monoclonal antibody, binds to receptor activator of nuclear factor $\kappa-\mathrm{B}$ ligand (RANKL) and prevents it to stir up, leading to diminished population and destructive properties of giant cells (11). Histopathologic analysis demonstrated the absence or decrease of at least $90 \%$ in the number of neoplastic giant cells and also an important decrease in tumoral stromal cells (11). There are reports demonstrating that neoadjuvant therapy with denosumab can make osteoclast-type giant cells disappear, both in the original tumor location and in its pulmonary metastasis (12). This medication may bring major changes to the therapeutic strategy of GCT in inaccessible anatomic locations such as spine or sacrum $(13,14)$. We believe that the major shortcoming of this study was its small sample size.

\section{Conclusion}

Considering the results of our study, we recommend resection of distal ulna for all GCTs in this anatomic location with grade 3 due to high local recurrence obtained 
with more conservative approaches. Curettage alone does not seem to be sufficient in this condition. Based on the functional results of our study and those of another case series on the resection of the distal ulna for tumors in that area, we do not recommend reconstruction after resection. However, extended curettage and bone grafting is an acceptable surgical method to treat grade 2 tumors. The corresponding author, on behalf of all authors, declares that there is no conflict of interest.

\section{Conflict of Interests}

The authors declare that they have no competing interests.

\section{References}

1. Amanatullah DF, Clark TR, Lopez MJ, Borys D, Tamurian RM. Giant cell tumor of bone. Orthopedics. 2014;37(2):112-20.

2. Vanni D, Pantalone A, Andreoli E, Caldora P, Salini V. Giant cell tumor of the distal ulna: a case report. J Med Case Rep. 2012;6(1):143.

3. Wada T, Kawai A, Ihara K, Sasaki M, Sonoda T, Imaeda T, et al. Construct validity of the Enneking score for measuring function in patients with malignant or aggressive benign tumours of the upper limb. Bone Joint J. 2007;89(5):659-63.

4. Ebrahimzadeh MH, Moradi A, Vahedi E, Kachooei AR, Birjandinejad A. Validity and reliability of the Persian version of shortened disabilities of the arm, shoulder and hand questionnaire (quick-DASH). International journal of preventive medicine. 2015;6.

5. Shin YH, Lee YH. Biomechanics of the Wrist. J Kore Fract Soc. 2016;29(1):93-100.

6. Minami A, Iwasaki N, Nishida K, Motomiya M, Yamada K, Momma D. Giant-cell tumor of the distal ulna treated by wide resection and ulnar support reconstruction: A case report. Case Report Med. 2010;2010.

7. Gracia I, Proubasta IR, Trullols L, Peiró A, Moya E, Cortés S, et al. Distal radioulnar joint prosthesis for the treatment of giant cell tumor of the distal ulna: a case report and literature review. Strateg Trauma Limb Reconstr. 2011;6(2):103-6.

8. Kotrych D, Żyluk A, Walaszek I, Bohatryrewicz A. Reconstruction of the distal radio-ulnar joint with a prosthesis of the distal ulna in the treatment of a recurrent giant cell tumour. Pol J Surg. 2011;83(9):51822.

9. Mariappan E, Mohanen P, Moses J. A Newer Technique of Distal Ulna Reconstruction Using Proximal Fibula and TFCC Reconstruction Using Palmaris Longus Tendon following Wide Resection of Giant Cell Tumour of Distal Ulna. Case Report Orthoped. 2013;2013.

10.Ferguson K, Jane M, Mahendra A. Giant cell tumour of the distal ulna: is reconstruction required after excision of the distal third of the ulna? J Hand Surg (Eur Vol). 2015:1753193415568912.

11.Branstetter DG, Nelson SD, Manivel JC, Blay J-Y, Chawla S, Thomas DM, et al. Denosumab induces tumor reduction and bone formation in patients with giant-cell tumor of bone. Clin Cancer Res. 2012;18(16):4415-24.

12.Yamagishi T, Kawashima H, Ogose A, Sasaki T, Hotta T, Inagawa $\mathrm{S}$, et al. Disappearance of giant cells and presence of newly formed bone in the pulmonary metastasis of a sacral giant?cell tumor following denosumab treatment: A case report. Oncol Let. 2016;11(1):243-6.

13.Mattei TA, Ramos E, Rehman AA, Shaw A, Patel SR, Mendel E. Sustained long-term complete regression of a giant cell tumor of the spine after treatment with denosumab. Spine J. 2014;14(7):e15-e21.

14.Mirzaei A, Bagherifard A, Bahrabadi M. Giant cell tumor of the sacrum: Series of 19 patients and review of the literature. Arch Bone Joint Surg. 2017. 